\title{
Relationship between the geometry patterns of vertebrobasilar artery and atherosclerosis
}

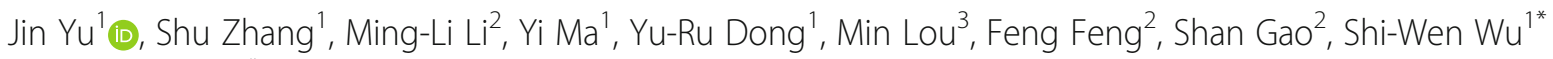
and Wei-Hai $\mathrm{Xu}^{2^{*}}$

\begin{abstract}
Background: The plaques at the dorsal or lateral wall of basilar artery (BA) are associated with pontine infarcts. We sought to explore the correlations between vertebrobasilar artery geometry and BA plaque locations.

Methods: We retrospectively analyzed the imaging and clinical data of 84 patients with BA atherosclerosis. On three-dimensional time-of-flight images, a side to side diameter difference of bilateral vertebral artery (VA) and BA bending were assessed. The vertebrobasilar artery geometry was qualitatively classified into four basic configurations: Walking, Tuning Fork, Dominant-Lambda, and Hypoplasia-Lambda. On high-resolution magnetic resonance imaging, the plaques were categorized based on the involvement of the ventral, dorsal, or lateral sides of BA wall. The relationships between vertebrobasilar artery geometry parameters and plaque locations were analyzed.

Results: Left VA dominance was identified in 28(33\%) patients, and right VA dominance in 22(26\%) patients. BA bending were detected in 49 patients. There were no significant correlations between the diameter difference/ratio of VA diameters and plaque locations, or between BA bending and plaque locations. BA plaques were evenly distributed in the vertebrobasilar arteries with Tuning Fork and Dominant-Lambda configurations. In Hypoplasia-Lambda group, however, plaques were more frequently located at the dorsal wall (58.57\%) than at the ventral (14.43\%) and lateral wall (26.71\%; $P=0.001)$. In Walking group, the plaques more likely occurred at the lateral (49.79\%) and dorsal (35.07\%) wall than at the ventral wall $(14.86 \%, P=0.02)$.

Conclusions: The geometric configurations of vertebrobasilar artery strongly influence the BA plaque locations. Further prospective studies are warranted to testify whether Hypoplasia-Lambda and Walking configurations are independent risk factors for pontine infarcts.
\end{abstract}

Keywords: Intracranial atherosclerosis, Vertebrobasilar system, Geometry, Plaque, Magnetic resonance imaging

\section{Background}

Vertebrobasilar atherosclerosis is a common cause of ischemic stroke. The underlying mechanisms include artery-to-artery embolism, in situ thrombo-occlusion, local branch occlusion, or hemodynamic impairment [1]. Local branch occlusion is the relatively more important

\footnotetext{
*Correspondence: wu_shiwen@yahoo.com; xuwh@pumch.cn

'Department of Neurology and Radiology, General Hospital of Chinese People's Armed Police Forces, 69 Yongding road, Haidian District, Beijing 100039, China

${ }^{2}$ Department of Neurology and Radiology, Peking Union Medical College Hospital, Chinese Academy of Medical Sciences, Shuaifuyuan 1, Dongcheng District, Beijing 100730, China

Full list of author information is available at the end of the article
}

mechanism, because the small and short perforating vessels of basilar artery (BA) are vulnerable to occlusion in the presence of parental artery atherothrombosis [1]. Recent in vivo studies have provided supportive evidence that the plaques at the dorsal or lateral wall of BA, where the branch arteries arise from, are associated with symptomatic pontine infarcts $[2,3]$.

It is known that mechanical and hemodynamic factors play a role in the development of atherosclerosis [4]. Early atherosclerotic plaques often develop at the sites with low or oscillatory wall shear stress [5], such as the inner wall of a curved artery, the outer wall of a bifurcation, and the apex of a junction. Unlike other systemic

(c) The Author(s). 2018 Open Access This article is distributed under the terms of the Creative Commons Attribution 4.0 International License (http://creativecommons.org/licenses/by/4.0/), which permits unrestricted use, distribution, and 
arteries with a tree-like branching pattern, the BA is the only artery in humans in which two flows merge. The diameters of the vertebral artery (VA) are of equal size in $6-38.5 \%$ of patients in angiographic or postmortem studies $[6,7]$. The variations of the geometry and asymmetrical inflow of vessels result in changes of the flow force distribution, that influenced the morphological deformation in the vertebrobasilar system (VBS) and induced infarcts in the different areas [8]. We hypothesized certain VBS geometry may be associate with certain distribution of plaque, which may influence the etiologies of infarction. In this study, using high-resolution magnetic resonance imaging (HR-MRI), we analyzed the relevance of the VBS geometry and BA plaque locations.

\section{Methods}

\section{Patients}

We retrospectively reviewed the HR-MRI databases (2008 to 2015) from three medical centers. All patients with BA atherosclerotic plaque on HR-MRI were enrolled if they fulfilled the following criteria: (1) had two normal VAs detected by magnetic resonance angiography (MRA); (2) image quality good enough for analysis. This observational study was approved by the local ethics committees. All the subjects signed an informed consent.

\section{Image analysis}

Details of our high-resolution MRI protocol were described elsewhere [3]. The images of BA wall were graded on a 3 -point scale: $1=$ nonvisualization, 2 = adequate, and $3=$ good. The images with a score of 2 or 3 were analyzed [8]. On MRA, the diameter of each VA was calculated as the average of the measurements made at three consecutive points, $3 \mathrm{~mm}$ apart, starting from the vertebrobasilar junction [9]. The dominant VA was defined if it was larger in diameter (difference $\geq 0.3 \mathrm{~mm}$ ) than the contralateral side [6]. The ratio of VA diameters was defined according the following formula: ratio = one side of diameter/the other side of larger diameter. A line was drawn between the top of the BA and junction of both VAs for reference to decide the side of BA bending [10].

Based on the TOF images, the VBS geometry was qualitatively classified into four basic geometric configurations: Walking, Tuning Fork, Dominant-Lambda, and Hypoplasia-Lambda (Fig. 1) [11]. The Walking geometry is distinguished by two equal VAs (diameter difference $<0.3 \mathrm{~mm}$ ) that bend in the same direction before merging into the BA [11]. The Tuning Fork configuration exhibits the two equal VAs that join at the confluence at rather symmetrical angles with respect to the BA, and the VAs bend in opposite directions [11]. In the Dominant-Lambda configuration, the $\mathrm{BA}$ path follows the direction dictated by the dominant VA, and the other VA, typically smaller, abuts the dominant VA

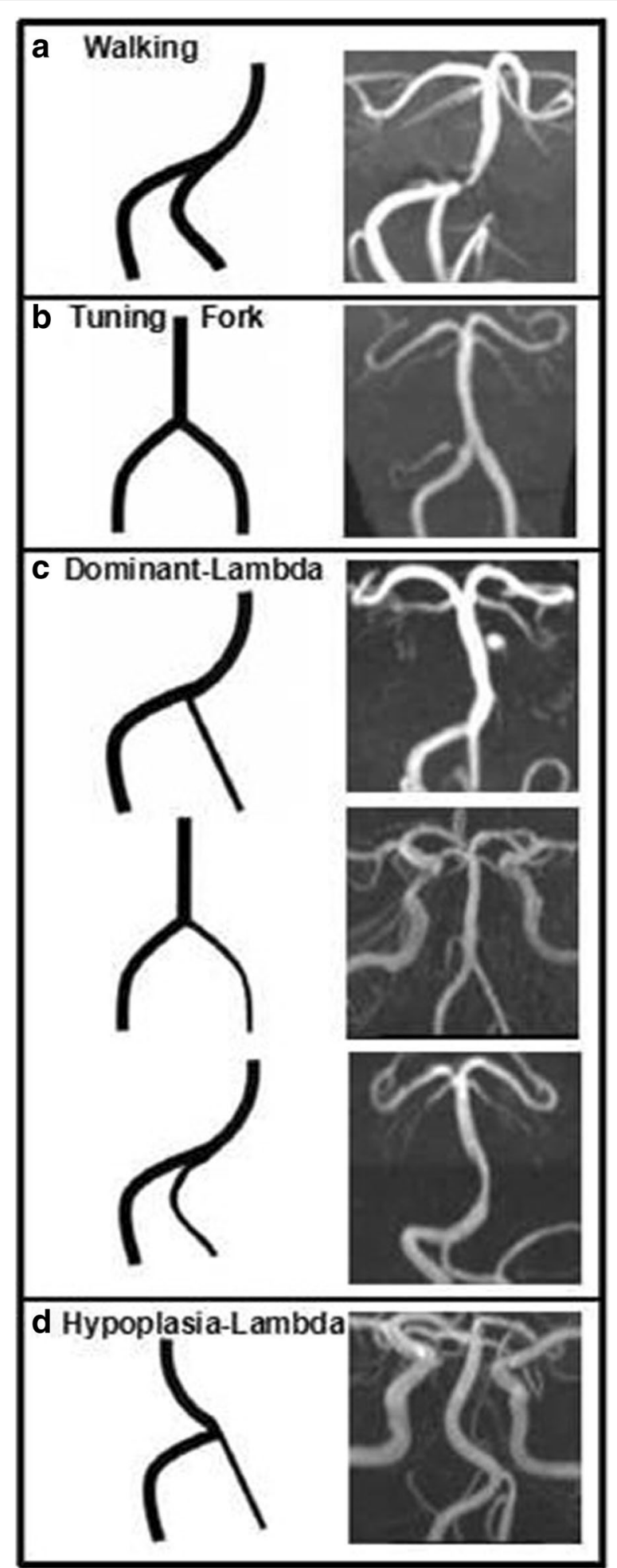

Fig. 1 Vertebrobasilar configurations. The vertebrobasilar system geometry is qualitatively classified into four basic geometric configurations: Walking (a), Tuning Fork (b), Dominant-Lambda (c), and Hypoplasia-Lambda (d). In each panel, a schematic of the configuration is followed by anterior-posterior magnetic resonance angiography 
or BA path, forming a pseudo T-junction [11]. In the Hypoplasia-Lambda configuration, the BA path follows the direction dictated by relatively hypoplastic VA.

Details of the design of plaque distribution have been published previously [3]. Briefly, all cross-sections with eccentric plaque were classified based on their plaque orientation being centered on the ventral, dorsal, and lateral (left or right) sides of the vessel. All images were reviewed by two experienced readers ( $\mathrm{Yu}$ and $\mathrm{Xu}$ ) blinded to clinical data, using software of the syngofast View-Viewer for DICOM images (Ver.1.0.0.34). The differences between the two observers were solved by consensus. VA diameters in the initial 10 patients were remeasured 2 months later for estimation of intraobserver and interobserver variability.

\section{Statistical analysis}

Intraobserver or interobserver variability for the measurements of VA was determined by intraclass correlation coefficient (ICC). Quantitative data are expressed as mean $\pm \mathrm{SD}$ and qualitative data are expressed as percentage. The continuous variables between the two groups were compared by the independent samples t-test. For each stenosis, the percentage of individual plaque distribution was calculated (Additional file 1: Table S1). The mean ventral, dorsal, lateral (left and right) plaque orientation of the total group was derived from the individual percentage distribution [12]. The comparison of the plaque distribution among different walls was performed by a Kruskal-Wallis test of the mean percentage of the distribution for each individual stenosis followed by Variance analysis and Bonferroni correction for multiple comparisons. Data comparisons among the four groups of vessels were conducted with the Wilcoxon test. Multiple correlation analysis between the ratio/difference of VA diameters and the percentage of individual plaque distribution from each patient were calculated. Correlation between the plaque distribution and the side of bending of the BA were assessed by multiple logistic regression analysis. A probability value of $<0.05$ was considered statistically significant.

\section{Results}

One hundred and nineteen patients with BA plaque were considered for enrollment. Five patients with poor image qualities and thirty patients with stenotic VAs were excluded. Eighty-four (mean age $62.4 \pm 12.9$ years, 61 male) patients were finally included for analysis. Forty-six patients $(54.8 \%)$ were symptomatic and new ischemic lesions were identified on diffusion-weighted imaging. Of the symptomatic patients, 29 had pontine infarctions, 1 had infarctions involving both pontine and extra-pontine area (with a cerebellum infarction), and 9 with extra-pontine infarctions (3 with thalamic infarction,
2 with occipital lobe infarction, 1 with cerebellum infarction, and 1 with medulla oblongata infarction). Eighteen patients (21.4\%) with BA atherosclerotic stenosis ( $\geq 50 \%$ ) were detected by MRA. There were $34(40.5 \%)$ patients with hypertension, 29(34.5\%) with hyperlipidemia, $20(23.8 \%)$ with diabetes mellitus, and 20(23.8\%) smoking patients.

The intraobserver variability was small for the measurements of VA (ICC 0.995, 95\% CI 0.980-0.999). The interobserver variability was also small for the measurements of VA (ICC 0.993, 95\% CI 0.972-0.998).

The average diameter of VA was $2.45 \pm 0.68 \mathrm{~mm}$ in the left and $2.35 \pm 0.52 \mathrm{~mm}$ in the right $(p=0.17)$. The diameters of the VA were of equal size in $34(40.5 \%)$ patients. Of the remaining 50 patients, the left VA dominance was identified in 28 (56\%) patients and the right VA dominance in 22 patients (44\%). The difference of VA diameters ranged from 0.0 to $1.8 \mathrm{~mm}$, and the ratio from 0.4 to 1.0. There were no significant correlations between the difference/ratio of VA diameters and the plaque locations (Multiple correlation analysis, Table 1). Forty-nine (58.3\%) patients had BA bending. In 50 patients with dominant VA, the BA was bent contrary to the dominant side in 18 (36\%), ipsilateral in $11(22 \%)$ and no bending in $21(42 \%)$. In 34 patients with equivalent VAs, the BA was bent laterally in $20(58.8 \%)$ and not bent in 14 (41.2\%). No significant correlation between the plaque locations and the side of BA bending (ventral: $p=0.759$, dorsal: $p=0.765$, left lateral: $p=0.763$, right lateral: $p=0.800$, Multiple logistic regression analysis).

BA plaques were evenly distributed in the vertebrobasilar arteries with Tuning Fork and Dominant-Lambda configurations (Table 2). However, in Hypoplasia-Lambda group, plaques were more frequently located at the dorsal wall $(58.57 \%)$ than at the ventral(14.43\%) and lateral wall (26.71\%; $P=0.001$, Variance analysis and Bonferroni correction test). In Walking group, the plaques more likely occurred at the lateral $(49.79 \%)$ and dorsal $(35.07 \%)$ wall than at the ventral wall $(14.86 \%, P=0.02$, Fig. 2$)$.

Table 1 Correlation analysis between the difference/ratio of vertebral artery diameters and basilar artery plaque locations

\begin{tabular}{|c|c|c|c|c|}
\hline & & Ventral wall & Dorsal wall & Lateral wall \\
\hline \multicolumn{5}{|l|}{ Difference } \\
\hline \multirow[t]{2}{*}{$<0.3 \mathrm{~mm}$} & $r$ & -0.292 & 0.250 & -0.018 \\
\hline & $p$ & 0.072 & 0.125 & 0.914 \\
\hline \multirow[t]{2}{*}{$\geq 0.3 \mathrm{~mm}$} & r & 0.076 & -0.102 & 0.138 \\
\hline & $p$ & 0.625 & 0.511 & 0.366 \\
\hline \multicolumn{5}{|l|}{ Ratio } \\
\hline & r & -0.038 & -0.001 & -0.010 \\
\hline & $p$ & 0.731 & 0.993 & 0.930 \\
\hline
\end{tabular}

Multiple correlation analysis with $\mathrm{p}$ and $\mathrm{r}$-values were given 
Table 2 Geometric configurations and basilar artery plaque locations

\begin{tabular}{lllll}
\hline Configuration & Ventral wall & Dorsal wall & Lateral wall & $P^{*}$ \\
\hline Walking (14) & $14.86 \%$ & $35.07 \%$ & $49.79 \%$ & $0.02^{1}$ \\
Tuning Fork (16) & $32.03 \%$ & $41.20 \%$ & $28.90 \%$ & 0.632 \\
Dominant-Lambda (40) & $24.48 \%$ & $40.03 \%$ & $35.09 \%$ & 0.083 \\
Hypoplasia-Lambda (14) & $14.43 \%$ & $58.57 \%$ & $26.71 \%$ & $0.001^{2}$ \\
$P$ & 0.268 & 0.291 & 0.220 & \\
\hline
\end{tabular}

$\mathrm{P}$ indicates comparisons in four groups, $P^{*}$ indicates comparisons in the ventral, dorsal, and lateral sides of BA wall

1 , ventral vs. Dorsal, $p=0.095$; ventral vs. Lateral, $p=0.005$; dorsal vs. lateral $p=0.220$

2 , ventral vs. Dorsal, $p=0.000$; ventral vs. Lateral, $p=0.284$; dorsal vs. lateral $p=0.008$

\section{Discussion}

In this study, we investigated the correlations between the vertebrobasilar artery geometry and BA plaque locations. It was observed that geometric configurations strongly influenced BA plaque locations. In the vetebrobasilar arteries with Hypoplasia-Lambda and Walking configurations,

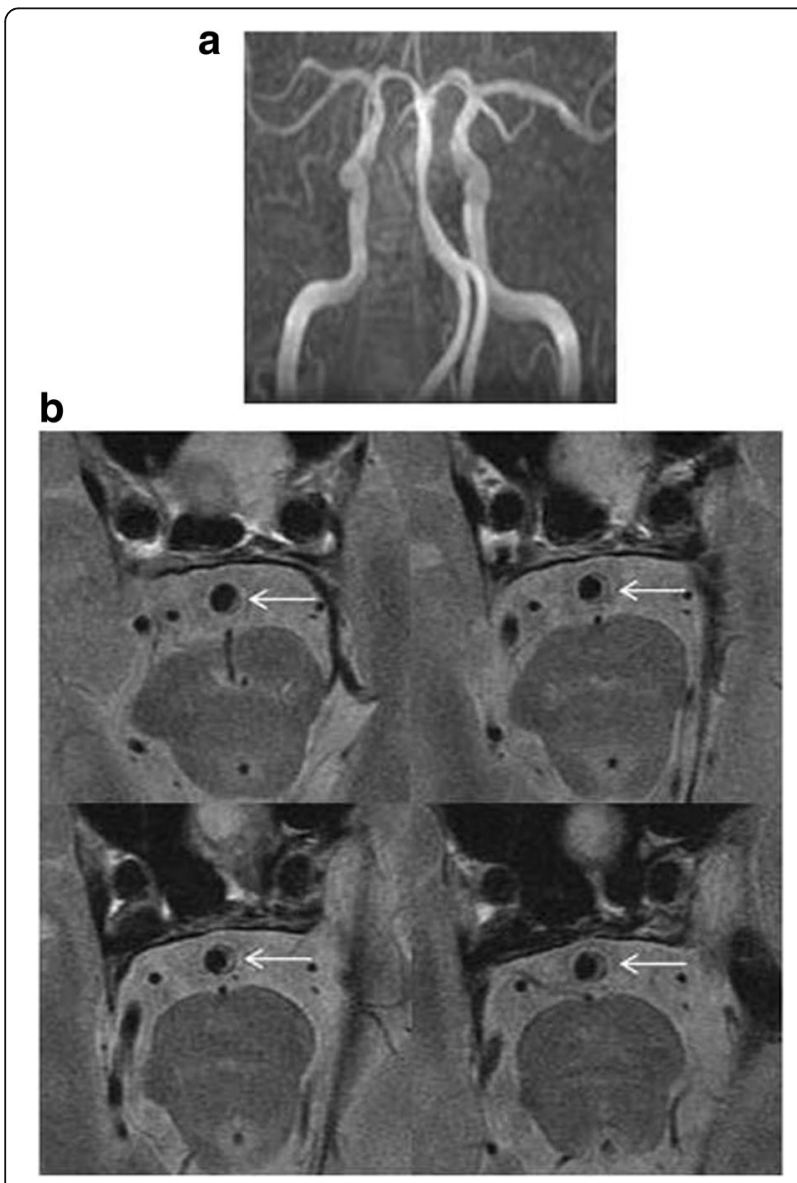

Fig. 2 Basilar artery atherosclerosis with Walking configuration. In a basilar artery with Walking configuration (a), atherosclerosis involving lateral wall (b) can be observed on 4 of 12 consecutive slices on high-resolution magnetic resonance imaging (arrows) the BA plaques more frequently occurred at the dorsal or the lateral wall, where the branches of BA mainly arise from. Theoretically, these two configurations may have a higher risk of pontine infarcts due to the mechanism of a parent artery plaque occluding a penetrating artery. The underlying hemodynamic mechanisms, however, remains elusive. Using MRI-Based models, Wake-Buck et al. analyzed the flow dynamics of five healthy subjects in vivo (2 with Walking geometry, 2 with Tuning Fork geometry and 1 with Dominant-Lambda) [11]. The BA geometry strongly influenced both skewing of the velocity profiles and the wall shear stress distributions in the VBS. Unfortunately, the detail of wall shear stress distribution in different geometry configurations was not reported. Another recent work found that the greatest BA-VA angle was observed in patients with posterior plaques and greatest mid-BA angles with lateral BA plaques [13]. We speculate that hemodynamic change occurs at the vertebrobasilar junction and at the mid-BA when the flow bends because of the mid-BA angle. According to our results, the low wall shear stress zones were assumed to be mainly distributed in the dorsal and lateral wall in the Hypoplasia-Lambda and Walking configurations. Further data from computerized hemodynamic studies are required to verify our hypothesis.

No correlation was observed between the ratio/difference of VA diameters and the plaque location. Actually, the asymmetric VAs had been regarded as a congenital variant, that don't result in a difference in the distribution of shear stress on the BA walls. Ravensbergen et al., by autopsy and a series of junction models, demonstrated that local regions of low wall shear stress (defined as wall shear stress $<1 \mathrm{~Pa}$ ) occurred at the crux of the junction and on the inner wall of the BA curve [14]. The difference of wall shear stress was not related to the VA dominance. On the other hand,similarly, no correlation was observed between the side of BA bending and plaque location. The meandering phenomenon of the $\mathrm{BA}$ is also viewed as a long-standing chronic process in adults. Clinical observations have linked these curving arteries to genetic defects, aging, atherosclerosis, hypertension and diabetes mellitus [15]. We consider both asymmetry of VA and BA bending may give a low contribution to the plaque locations.

Our study suffered from several limitations. First, in this study, only $21 \%$ patients had a severe stenosis $(\geq 50 \%)$. Our results are not appropriate for advanced BA atherosclerosis which is often associated with embolic stroke. Second, patients with any VA stenosis were excluded from the study. Because VA is the upstream vessel of BA, it is not known the hemodynamic effects of VA stenosis to BA atherosclerosis. The hemodynamic roles of VA to BA in our study may be simplified and underestimated. Third, the sample of our study was too small 
to analyze the relationships between the four configurations and clinical presentations.

\section{Conclusion}

Despite of these limitations, our results may be of clinical importance. In practice, patients with intracranial atherosclerosis may be exposed to similar stroke risk factors but have heterogeneous clinical outcomes. A precise stroke risk stratification for an individual patient is not available. Our results provide a potentially new research direction for primary and secondary prevention of cerebrovascular diseases, that individual intracranial artery geometry should be analyzed in addition to traditional risk factors. Further prospective studies are warranted to testify whether Hypoplasia-Lambda and Walking configurations are independent risk factors for pontine infarcts.

\section{Additional file}

Additional file 1: Table S1: Percentage of individual plaque distribution. Calculations of the percentage of individual plaque distribution: The quadrant grouping slice is patient specific. For each stenosis, the percentage of individual plaque distribution was calculated. If a stenosis only had one slice with ventral plaque, for example, the percentage of its individual plaque distribution is ventral $100 \%$, dorsal $0 \%$, lateral $0 \%$. If the stenosis have 3 slices (ventral 2, dorsal 1), the percentage is ventral 66\%, dorsal 33\%, lateral 0\%. (DOCX $18 \mathrm{~kb})$

\section{Abbreviations}

3D TOF: 3 Dimensional time-of-flight; BA: Basilar artery; FOV: Field of view; HR-MRI: High-resolution magnetic resonance imaging; MRA: Magnetic resonance angiography; PDWl: Proton density weighted imaging; T1WI: T1 weighted imaging; T2WI: T2 weighted imaging; VA: Vertebral artery; VBS: Vertebrobasilar system

\section{Funding}

This study is supported by Program for National Natural Science Foundation of China (81471207 and 81671370) and 2016 Peking Union Medical College Hospital science fund for junior faculty.

\section{Authors' contributions}

XW and WS participated in the design and coordination of the study and has been involved in revising the manuscript for important intellectual content. YJ performed MRI data and statistical analysis, drafted the manuscript. ZS, LM and GS participated in the clinical evaluation of the patients, performed MRI data analysis and interpretation. LML, MY, DY and FF carried out the MRI acquisition. All authors read and approved the final manuscript.

\section{Ethics approval and consent to participate}

This observational study was approved by the ethics committee at Peking Union Medical College Hospital, General Hospital of Chinese People's Armed Police Forces, and Zhejiang University 2nd affiliate hospital. All patients gave written informed consent.

\section{Competing interests}

The authors declare that they have no competing interests.

\section{Publisher's Note}

Springer Nature remains neutral with regard to jurisdictional claims in published maps and institutional affiliations.

\section{Author details}

'Department of Neurology and Radiology, General Hospital of Chinese People's Armed Police Forces, 69 Yongding road, Haidian District, Beijing
100039, China. ${ }^{2}$ Department of Neurology and Radiology, Peking Union Medical College Hospital, Chinese Academy of Medical Sciences, Shuaifuyuan 1, Dongcheng District, Beijing 100730, China. ${ }^{3}$ Department of Neurology, Zhejiang University 2nd Affiliate Hospital, Hangzhou, Zhejiang Province, China.

Received: 27 December 2017 Accepted: 30 May 2018

Published online: 12 June 2018

\section{References}

1. Kim JS, Nah HW, Park SM, Kim SK, Cho KH, Lee J, et al. Risk factors and stroke mechanisms in atherosclerotic stroke: intracranial compared with extracranial and anterior compared with posterior circulation disease. Stroke. 2012;43:3313-8

2. Klein IF, Lavallée PC, Mazighi M, Schouman-Claeys E, Labreuche J, Amarenco P. Basilar artery atherosclerotic plaques in paramedian and lacunar pontine infarctions: a high-resolution MRI study. Stroke. 2010;41:1405-9.

3. Yu J, Li ML, Xu YY, Wu SW, Lou M, Mu XT, et al. Plaque distribution of low-grade basilar artery atherosclerosis and its clinical relevance. BMC Neurol. 2017:17:8.

4. Ravensbergen J, Ravensbergen JW, JKB K, Hillen B, Hoogstraten HW. Localizing role of hemodynamics in atherosclerosis in several human vertebrobasilar junction geometries. ArteriosclThrom Vas. 1998;18:708-16.

5. Asakura T, Karino T. Flow patterns and spatial distribution of atherosclerotic lesions in human coronary arteries. Circ Res. 1990;66:1045-66.

6. Jeng JS, Yip PK. Evaluation of vertebral artery hypoplasia and asymmetry by colorcoded duplex ultrasonography. Ultrasound Med Biol. 2004;30:605-9.

7. Cagnie B, Petrovic M, Voet D, Barbaix E, Cambier D. Vertebral artery dominance and hand preference: is there a correlation? Man Ther. 2006;11:153-6.

8. Ma N, Jiang WJ, Lou X, Ma L, Du B, Cai JF. etal. Arterial remodeling of advanced basilar atherosclerosis: a 3-tesla MRI study. Neurology. 2010;75:253-8.

9. Hong JM, Chung CS, Bang OY, Yong SW, Joo IS, Huh K. Vertebral artery dominance contributes to basilar artery curvature and peri-vertebrobasilar junctional infarcts. J Neurol Neurosurg Psychiatry. 2009;80:1087-92.

10. Nishikata M, Hirashima Y, Tomita T, Futatsuya R, Horie Y, Endo S. Measurement of basilar artery bending and elongation by magnetic resonance cerebral angiography: relationship to age, sex and vertebral artery dominance. Arch Gerontol Geriatr. 2004;38:251-9.

11. Wake-Buck AK, Gatenby JC, Gore JC. Hemodynamic characteristics of the vertebrobasilar system analyzed using MRI-based models. PLoS One. 2012; 7:e51346.

12. Xu WH, Li ML, Gao S, Ni J, Zhou LX, Yao M, et al. Plaque distribution of stenotic middle cerebral artery and its clinicalrelevance. Stroke. 2011;42:2957-9.

13. Kim BJ, Lee KM, Kim HY, Kim YS, Koh SH, Heo SH, et al. Basilar artery plaque and pontine infarction location and vascular geometry. J Stroke. 2018;20:92-8.

14. Ravensbergen J, Krijger JK, Hillen B, Hoogstraten HW. The influence of the angle of confluence on the flow in a vertebro-basilar junction model. J Biomech. 1996:29:281-99.

15. Han HC. Twisted blood vessels: symptoms, etiology and biomechanical mechanisms. J Vasc Res. 2012;49:185-97.
Ready to submit your research? Choose BMC and benefit from:

- fast, convenient online submission

- thorough peer review by experienced researchers in your field

- rapid publication on acceptance

- support for research data, including large and complex data types

- gold Open Access which fosters wider collaboration and increased citations

- maximum visibility for your research: over $100 \mathrm{M}$ website views per year

At BMC, research is always in progress.

Learn more biomedcentral.com/submissions 